\title{
AN ELEMENTARY SIMULTANEOUS APPROXIMATION THEOREM
}

\author{
THEODORE KILGORE
}

(Communicated by J. Marshall Ash)

\begin{abstract}
We will give an elementary and direct proof that for $f \in C^{q}[-1,1]$ there exists a sequence of polynomials $P_{n}$ of degree at most $n(n>2 q)$ such
\end{abstract} that for $k=0, \ldots, q$

$$
\left|f^{(k)}(x)-P_{n}^{(k)}(x)\right| \leq M_{q, k}\left(\frac{\sqrt{1-x^{2}}}{n}\right)^{q-k} E_{n-q}\left(f^{(q)}\right),
$$

with $M_{q, k}$ depending only upon $q$ and $k$. Moreover $f^{(q)}( \pm 1)=P_{n}^{(q)}( \pm 1)$.

\section{INTRODUCTION}

We will prove here the following

Theorem. Let $f \in C^{q}[-1,1]$. Then there exists a sequence of polynomials $P_{n}$ of degree at most $n(n>2 q)$ such that for $k=0, \ldots, q$

$$
\left|f^{(k)}(x)-P_{n}^{(k)}(x)\right| \leq M_{q, k}\left(\sqrt{1-x^{2}} / n\right)^{q-k} E_{n-q}\left(f^{(q)}\right),
$$

where the constants $M_{q, k}$ depend only on $q$ and $k$. Moreover, $f^{(q)}( \pm 1)=$ $P_{n}^{(q)}( \pm 1)$.

Notation. Most of our notation is quite standard and will be introduced in context. As the first instance, we write $C^{q}[-1,1]$ for the space of $q$ times continuously differentiable functions, and

$$
E_{n}(f):=\inf _{\text {degree } p_{n} \leq n}\left\|f-p_{n}\right\|,
$$

in which the norm is the usual supremum norm.

Context and antecedents. Those results that give pointwise estimates for polynomial approximation and/or establish sharp rates for simultaneous approximation of derivatives are often referred to as "of Timan type." The main feature of our theorem is a new and simply motivated proof directly employing basic results on approximation by trigonometric polynomials; its precise statement is also new. For comparison we state some of the better known previous results, introducing for brevity the notation

$$
\Delta_{n}(x):=\frac{1}{n^{2}}+\frac{\sqrt{1-x^{2}}}{n} .
$$

Received by the editors May 9, 1991 and, in revised form, October 10, 1991.

1991 Mathematics Subject Classification. Primary 41A28. 
Timan [11] showed the following:

Let $f \in C^{q}[-1,1]$. Then there is a sequence of polynomials $P_{n}$ of degree at most $n$ such that

$$
\left|f(x)-P_{n}(x)\right| \leq M\left(\Delta_{n}(x)\right)^{q} \omega\left(f^{(q)} ; \Delta_{n}(x)\right) .
$$

The notation $\omega(f ; h)$ (modulus of continuity) is defined by

$$
\omega(f ; h):=\sup _{|x-y| \leq h}|f(x)-f(y)| .
$$

Telyakovskii [10] improved Timan's result by replacing $\Delta_{n}(x)$ with $\sqrt{1-x^{2}} / n$. Trigub [12] established the simultaneous approximation result that for $k=$ $0, \ldots, q$

$$
\left|f^{(k)}(x)-P_{n}^{(k)}(x)\right| \leq M\left(\Delta_{n}(x)\right)^{q-k} \omega\left(f^{(q)} ; \Delta_{n}(x)\right) .
$$

Gopengauz [6] replaced $\Delta_{n}(x)$ (in both locations) in Trigub's result with $\sqrt{1-x^{2}} / n$, and Leviatan [8] replaced the modulus of continuity in Trigub's result with $E_{n-q}\left(f^{(q)}\right)$. Our result combines features of the theorems of Gopengauz and Leviatan, and that of Leviatan follows in particular as a corollary from our theorem. Thus, the "interpolatory" result of Balázs, Kilgore, and Vértesi [2], useful in formulating estimates for simultaneous approximation via interpolation, also follows from the present theorem. More recently, the author stated and proved a version of this same theorem for the case $q=1$ in Kilgore [7], which also surveys some recent applications to interpolation.

\section{METHOD OF PROOF}

Our theorem will follow from two basic results on trigonometric best approximation of $2 \pi$-periodic functions (Lemma 1 and Lemma 2 ) and from several observations on derivatives (Lemmas $3,4,5$ ). To avoid a convoluted presentation, we will not actually estimate the constants $M_{q, k}$, but it should be clear that reasonable estimates can be obtained, especially for small $q$. The estimates can be improved if the conclusion $f^{(q)}( \pm 1)=P_{n}^{(1)}( \pm 1)$ is dropped.

Before embarking on the sequence Lemmas $1-5$, we introduce the notation

$$
E_{n}^{*}(f):=\inf _{\text {order of } T_{n} \leq n}\left\|f-T_{n}\right\|,
$$

in which $f$ is a $2 \pi$-periodic function and $T_{n}$ is a trigonometric polynomial (linear combination of $1, \cos \theta, \ldots, \cos n \theta, \sin \theta, \ldots, \sin n \theta$ ). The order of $T_{n}$ signifies the highest multiple of $\theta$ appearing in the actual expansion of $T_{n}$.

Lemma 1. Let $f$ be a $k$ times differentiable periodic function. Then there exists for $k=0,1,2, \ldots$ a constant $\alpha_{k} \leq(\pi / 2)^{2}$ independent of $f$ and $n$ such that

$$
E_{n}^{*}(f) \leq \alpha_{k}(n+1)^{-k} E_{n}^{*}\left(f^{(k)}\right) .
$$

Proof. This is a well-known result and is sufficient for our purposes. For a quite simple and basic proof one may consult Cheney [3]. In fact, $\alpha_{k} \leq \pi / 2$ for all $k$ is known. The best possible values are estimated in Favard [5] or Achieser and Krein [1].

Our next lemma appeared in Czipszer and Freud [4], using as a proof the listed properties of the de la Vallee-Poussin means of the Fourier expansion, 
which are simply and succinctly demonstrated among other places in the text of Lorentz [9]. We prove the lemma for completeness and to highlight the independence of our theorem from the other results mentioned in the introduction.

Lemma 2. Let $f$ be a $2 \pi$-periodic function that is $k$ times continuously differentiable. Let $T_{n}(\theta)$ be a trigonometric polynomial of order at most $n$ satisfying for some constant $C$ the inequality

$$
\left\|f(\theta)-T_{n}(\theta)\right\| \leq C E_{n}^{*}(f) .
$$

Then there exists a constant $\beta_{k}$ independent of $f$ and $n$ such that

$$
\left\|f^{(k)}(\theta)-T_{n}^{(k)}(\theta)\right\| \leq \beta_{k} C E_{n}^{*}\left(f^{(k)}\right) .
$$

Proof. Let $s_{j} f$ denote the Fourier expansion of $f$, truncated after order $j$. The de la Vallée-Poussin means are the linear operators $V_{n} f$ defined for $n=$ $1,2, \ldots$ by

$$
V_{n} f:=\frac{1}{n} \sum_{j=n}^{2 n-1} s_{j} f
$$

One has $V_{n}\left(f^{\prime}\right)=\left(V_{n} f\right)^{\prime},\left\|V_{n}\right\| \leq 3$ for $n=1,2, \ldots$, and $V_{n}\left(T_{n}\right)=T_{n}$ for order $T_{n} \leq n$. Immediately

$$
\begin{aligned}
\left\|f^{\prime}-T_{n}^{\prime}\right\| & \leq\left\|f^{\prime}-V_{n}\left(f^{\prime}\right)\right\|+\left\|\left(V_{n} f\right)^{\prime}-T_{n}^{\prime}\right\| \\
& \leq\left(1+\left\|V_{n}\right\|\right) E_{n}^{*}\left(f^{\prime}\right)+2 n\left\|V_{n}(f)-T_{n}\right\| \\
& \leq 4 E_{n}^{*}\left(f^{\prime}\right)+2 n\left\|V_{n}\right\|\left\|f-T_{n}\right\| \\
& \leq 4 E_{n}^{*}\left(f^{\prime}\right)+6 C n E_{n}^{*}(f) \leq 4 E_{n}^{*}\left(f^{\prime}\right)+3 C \pi E_{n}^{*}\left(f^{\prime}\right),
\end{aligned}
$$

using the listed properties of $V_{n}$, the Bernstein inequality, and Lemma 1. The lemma is thus demonstrated for $k=1$ and can obviously be established by repetition for other values of $k$.

Another estimate for the constants $\beta_{k}$ is found in the cited article of Czipszer and Freud, of the $\beta_{k} \leq A \log [\min (n, k)+1]$, where the constant $A$ is independent of $n, f$, and $k$.

Lemma 3. Let $h(\theta)$ be a $2 \pi$-periodic function that is $q$ times continuously differentiable. Then for each $n>q$ there exists a trigonometric polynomial $T_{n}(\theta)$ of order at most $n$ and constants $\gamma_{0}, \ldots, \gamma_{q}$ independent of $h$ and $n$ such that for $k=0, \ldots, q$

(a) $\left\|h^{(k)}(\theta)-T_{n}^{(k)}(\theta)\right\| \leq\left(\gamma_{k} / n^{q-k}\right) E_{n}^{*}\left(h^{(q)}\right)$,

(b) $h^{(k)}(0)-T_{n}^{(k)}(0)=h^{(k)}(\pi)-T_{n}^{(k)}(\pi)=0$,

and $T_{n}$ is even if $h$ is even, odd if $h$ is odd.

Proof. For $q>0$ let the integer $m$ be chosen as the greatest integer in $(n-1) / q$. Let $B_{n}(\theta)$ be a best approximation for $h$. Then (a) holds by Lemma 2 combined with Lemma 1.

We now define polynomials $T_{n, 0}(\theta), \ldots, T_{n, q}(\theta)$ by the following procedure:

$$
T_{n, 0}(\theta):=B_{n}(\theta)+\left(h(0)-B_{n}(0)\right)\left(\frac{1+\cos \theta}{2}\right)+\left(h(\pi)-B_{n}(\pi)\right)\left(\frac{1-\cos \theta}{2}\right),
$$


and we note that $T_{n, 0}(\theta)$ satisfies conclusion (a) with appropriate constants $\gamma_{k}^{(0)}$ for $k=0, \ldots, q$. Moreover, $T_{n, 0}$ satisfies (b) for the value $k=0$. We now define $T_{n, k}$ for $k=1, \ldots, q$ in recursive fashion by

$$
\begin{aligned}
T_{n, k}(\theta):=T_{n, k-1}(\theta)+ & \left(h^{(k)}(0)-T_{n, k-1}^{(k)}(0)\left(\frac{1+\cos \theta}{2}\right)\right. \\
& \left.+(-1)^{k m}\left(h^{(k)}(\pi)-T_{n, k-1}^{(k)}(\pi)\right)\left(\frac{1-\cos \theta}{2}\right)\right) \frac{\sin ^{k} m \theta}{k ! m^{k}} .
\end{aligned}
$$

We note first of all that $T_{n, k}$ satisfies (b) for derivatives from 0 up to $k$, and thus $T_{n, q}$ satisfies (b) of the lemma for derivatives 0 to $q$. We will define $T_{n}(\theta):=T_{n, q}(\theta)$. Lemma 2 suffices for the estimates required in (a), providing for each $k=1, \ldots, q$ a set of constants $\gamma_{0}^{(k)}, \ldots, \gamma_{q}^{(k)}$ such that

$$
\left\|h^{(j)}(\theta)-T_{n, k}^{(j)}(\theta)\right\| \leq\left(\gamma_{j}^{(k)} / n^{q-j}\right) E_{n}^{*}\left(h^{(q)}\right), \quad j=0, \ldots, q .
$$

To establish that $T_{n}(\theta)$ may be chosen even if $h(\theta)$ is even and odd if $h(\theta)$ is odd, it suffices to note that $B_{n}(\theta)$ may be chosen even if $h$ is even or odd if $h$ is odd, and the construction of the polynomials $T_{n, k}$ preserves evenness or oddness. Moreover, if $h$ is odd, then $T_{n, 0}=B_{n} ; T_{n, 2}=T_{n, 1} ; T_{n, 4}=T_{n, 3}$, and so on, and if $h$ is even, then $T_{n, 1}=T_{n, 0} ; T_{n, 3}=T_{n, 2} ; T_{n, 5}=T_{n, 4}$, and so on up to $T_{n, q}$.

Lemma 4. Let $\left\{H_{n}(\theta)\right\}$ be a sequence of $q$ times continuously differentiable $2 \pi$-periodic functions satisfying

$$
H_{n}(\theta) / \sin ^{q} \theta \rightarrow 0 \text { as } \theta \rightarrow 0 \text { or } \theta \rightarrow \pi
$$

and satisfying for some sequence of nonnegative numbers $\left\{\varepsilon_{n}\right\}$ the inequalities $\left\|H_{n}^{(k)}(\theta)\right\| \leq C / n^{q-k} \varepsilon_{n}$ for $k=0, \ldots, q$. Then for $k=0, \ldots, q$ the functions $H_{n, k}(\theta)$ satisfy $\left\|H_{n, k}(\theta)\right\| \leq C K / n^{q-k} \varepsilon_{n}$ with $K$ dependent only on $q$ (and $k)$, where $H_{n, 0}:=H_{n}$, and for $k=0, \ldots, q-1$

$$
H_{n, k+1}(\theta):=H_{n, k}^{\prime}(\theta)+(q-k)\left(\cos \theta H_{n, k}(\theta)\right) / \sin \theta .
$$

Proof. We will use induction and suppose that the lemma is true for all integers $q$ less than the given one, noting that the conclusion is vacuously true if $q=0$.

We have

$$
H_{n, 1}(\theta)=H_{n, 0}^{\prime}(\theta)+q \frac{\cos \theta H_{n, 0}(\theta)}{\sin \theta}=H_{n}^{\prime}(\theta)+q \cos \theta \frac{H_{n}(\theta)}{\sin \theta},
$$

in which the magnitude of the second term may be estimated by Rolle's Theorem. We obtain, since $|(\theta \cos \theta) / \sin \theta|<1$ for $|\theta| \leq \pi / 2$, with a similar estimate if $|\theta-\pi| \leq \pi / 2$,

$$
\left\|H_{n, 1}(\theta)\right\| \leq(1+q)\left\|H_{n}^{\prime}(\theta)\right\| \leq C(q+1) \varepsilon_{n} / n^{q-1} .
$$

Moreover,

$$
\frac{H_{n, q}(\theta)}{\sin ^{q-1} \theta}=\frac{H_{n}^{\prime}(\theta)}{\sin ^{q-1} \theta}+\frac{H_{n}(\theta)}{\sin ^{q} \theta} \cdot q \cos \theta,
$$

and therefore $H_{n, 1}(\theta) / \sin ^{q-1} \theta \rightarrow 0$ as $\theta \rightarrow 0, \pi$. And clearly $H_{n, 1}(\theta)$ is $q-1$ times continuously differentiable. 
The conclusion of the lemma will hold for $H_{n, 1}$ provided that we show the existence of a $K_{1}$ such that for $k=0, \ldots, q-1$

$$
\left\|H_{n, 1}^{(k)}(\theta)\right\| \leq \frac{C \cdot K_{1} \varepsilon_{n}}{n^{q-k-1}} .
$$

To this end we note that

$$
H_{n, 1}^{(k)}(\theta)=H_{n, 0}^{(k+1)}(\theta)+q\left(\frac{\cos \theta H_{n, 0}(\theta)}{\sin \theta}\right)^{(k)},
$$

and that only the magnitude of the second term requires further investigation. We have for arbitrary $\theta$

$$
\begin{aligned}
\left|\left(\frac{\cos \theta H_{n, 0}(\theta)}{\sin \theta}\right)^{(k)}\right| & \leq \sum_{j=0}^{k}\left(\begin{array}{c}
k \\
j
\end{array}\right)\left|(\cos \theta)^{(k-j)}\right| \cdot\left|\left(\frac{H_{n}(\theta)}{\sin \theta}\right)^{(j)}\right| \\
& \leq \sum_{j=0}^{k}\left(\begin{array}{c}
k \\
j
\end{array}\right)\left|\left(\frac{H_{n}(\theta)}{\sin \theta}\right)^{(j)}\right| .
\end{aligned}
$$

In turn,

$$
\begin{aligned}
\left|\left(\frac{H_{n}(\theta)}{\sin \theta}\right)^{(j)}\right| & \leq \sum_{l=0}^{j}\left(\begin{array}{l}
j \\
l
\end{array}\right) \mid\left(H_{n}^{(l)}(\theta)||\left(\frac{1}{\sin \theta}\right)^{(j-l)}\left|\leq \sum_{l=0}^{j}\left(\begin{array}{l}
j \\
l
\end{array}\right)(j-l) !\right| \frac{H_{n}^{(l)}(\theta)}{\sin ^{j-l+1} \theta} \mid\right. \\
& \leq \frac{1}{j+1} \sum_{l=0}^{j}\left(\begin{array}{c}
j+1 \\
l
\end{array}\right)\left(\frac{\pi}{2}\right)^{j-l+1}\left\|H_{n}^{(j+1)}(\theta)\right\| \leq \frac{C \cdot C^{\prime}}{n^{q-j-1}} \varepsilon_{n},
\end{aligned}
$$

for some constant $C^{\prime}$.

Now we get

$$
\left\|H_{n, 1}^{(k)}(\theta)\right\| \leq \frac{C \varepsilon_{n}}{n^{q-k-1}}+q \sum_{j=0}^{k}\left(\begin{array}{c}
k \\
j
\end{array}\right) \frac{C \cdot C^{\prime}}{n^{q-j-1}} \varepsilon_{n} \leq \frac{C \cdot K_{1}}{n^{q-k-1}} \varepsilon_{n},
$$

and the lemma holds with the function $H_{n, 1}$ in place of the function $H_{n}$. Hence, there is a constant $K_{2}$ such that for $k=2, \ldots, q$ (shifting indices)

$$
\left\|H_{n, k}\right\| \leq \frac{C \cdot K_{2} \varepsilon_{n}}{n^{q-k}}
$$

and we have already seen that

$$
\left\|H_{n, 1}\right\| \leq \frac{C(q+1)}{n^{q-1}} \varepsilon_{n} .
$$

Therefore the lemma holds for $H_{n}$ with constant $K=\max \left\{q+1, K_{2}\right\}$.

Lemma 5. Let $G \in C^{q}[-1,1]$ be such that $G^{(k)}( \pm 1)=0$ for $k=0, \ldots, q$, and $H(\theta):=(G(\cos \theta)) / \sin ^{q}(\theta)$. Then for $K_{q}$ depending only upon $q$

$$
E_{n-q}^{*}\left(D_{\theta}^{q} H\right) \leq K_{q} E_{n-q}\left(D_{x}^{q} G\right) .
$$

Proof. The lemma is obvious if $q=0$. We assume that the lemma holds true for all values of $q$ less than the given one. We note that

$$
D_{\theta} H(\theta)=\frac{-1}{\sin ^{q-1} \theta}\left(G^{\prime}(\cos \theta)+q \cos \theta \frac{G(\cos \theta)}{1-\cos ^{2} \theta}\right) .
$$


With $x=\cos \theta$, the expression in parentheses is a function of $x$ that lies in $C^{q-1}[-1,1]$ and satisfies

$$
\left(G^{\prime}(x)+\frac{q x G(x)}{1-x^{2}}\right)^{(k)}=0, \quad x= \pm 1, k=0, \ldots, q-1 .
$$

Therefore, in accordance with our assumption that the lemma operates for $q-1$, we have

$$
\begin{aligned}
E_{n-q}^{*}\left(D_{\theta} H\right) & =E_{n-q}^{*}\left(D_{\theta}^{q-1}\left(D_{\theta} H\right)\right) \leq K_{q-1} E_{n-q}\left(D_{x}^{q-1}\left(G^{\prime}(x)+\frac{q x G(x)}{1-x^{2}}\right)\right) \\
& \leq K_{q-1}\left(E_{n-q}\left(D_{x}^{q} G(x)\right)+q E_{n-q}\left(D_{x}^{q-1}\left(\frac{x G(x)}{1-x^{2}}\right)\right)\right) .
\end{aligned}
$$

Our proof is now completed by showing that

$$
E_{n-q}\left(D_{x}^{q-1}\left(\frac{x G(x)}{1-x^{2}}\right)\right) \leq\left(2^{q}-1\right) E_{n-q}\left(D_{x}^{q} G(x)\right),
$$

from which it would clearly follow that $K_{q} \leq\left(2^{q}\right) K_{q-1}$. We have for $x \in$ $[-1,1]$ that

$$
\begin{aligned}
\left(\frac{x G(x)}{1-x^{2}}\right)^{(q-1)}= & \sum_{k=0}^{q-1}\left(\begin{array}{c}
q-1 \\
k
\end{array}\right) G^{(q-1-k)}(x)\left(\frac{x}{1-x^{2}}\right)^{(k)} \\
= & \frac{1}{2} \sum_{k=0}^{q-1}\left(\begin{array}{c}
q-1 \\
k
\end{array}\right) G^{(q-1-k)}(x)\left(\frac{1}{1-x}\right)^{(k)} \\
& -\frac{1}{2} \sum_{k=0}^{q-1}\left(\begin{array}{c}
q-1 \\
k
\end{array}\right) G^{(q-1-k)}(x)\left(\frac{1}{1+x}\right)^{(k)} \\
= & \frac{1}{2} \sum_{k=0}^{q-1}\left(\begin{array}{c}
q-1 \\
k
\end{array}\right) k ! \frac{G^{(q-1-k)}(x)}{(1-x)^{k+1}} \\
& +\frac{1}{2} \sum_{k=0}^{q-1}(-1)^{k+1}\left(\begin{array}{c}
q-1 \\
k
\end{array}\right) k ! \frac{G^{(q-1-k)}(x)}{(1+x)^{k+1}} .
\end{aligned}
$$

Therefore,

$$
\begin{aligned}
E_{n-q} & \left(\left(\frac{x G(x)}{1-x^{2}}\right)^{(q-1)}\right) \\
\leq & \frac{1}{2} \sum_{k=0}^{q-1}\left(\begin{array}{c}
q-1 \\
k
\end{array}\right) k !\left[E_{n-q}\left(\frac{G^{(q-1-k)}(x)}{(1-x)^{k+1}}\right)+E_{n-q}\left(\frac{G^{(q-1-k)}(x)}{(1-x)^{k+1}}\right)\right] .
\end{aligned}
$$

We may examine for arbitrary $k$ the expression

$$
E_{n-q}\left(\frac{G^{(q-1-k)}(x)}{(1-x)^{k+1}}\right)
$$

and estimate its magnitude. Let $P_{n-q}^{*}(x)$ be any polynomial of degree $n-q$ or less approximating the function $G^{(q-1-k)}(x) /(1-x)^{k+1}$. Using repeatedly 
Cauchy's mean value theorem, we obtain

$$
\begin{aligned}
\frac{G^{(q-1-k)}(x)}{(1-x)^{k+1}}-P_{n-q}^{*}(x) & =\frac{G^{(q-k-1)}(x)-(1-x)^{k+1} P_{n-q}^{*}(x)}{(1-x)^{k+1}} \\
& =\frac{1}{(k+1) !}\left(G^{(q)}(y)-\left[(1-y)^{k+1} P_{n-q}^{*}(y)\right]^{(k+1)}\right)
\end{aligned}
$$

for some point $y$ between $x$ and 1. Furthermore, if $Q_{n-q}(x)$ is a polynomial of best approximation of degree at most $n-q$ for $G^{(q)}(x)$, then we are free to choose $P_{n-q}^{*}(x)$ of degree $n-q$ or less such that

$$
\left[(1-x)^{k+1} P_{n-q}^{*}(x)\right]^{(k+1)}=Q_{n-q}(x) .
$$

Using this particular choice for $P_{n-q}^{*}$, we obtain

$$
E_{n-q}\left(\frac{G^{(q-k-1)}(x)}{(1-x)^{k+1}}\right) \leq\left\|\frac{G^{(q-k-1)}(x)}{(1-x)^{k+1}}-P_{n-q}^{*}(x)\right\| \leq \frac{1}{(k+1) !} E_{n-q}\left(G^{(q)}\right) .
$$

Similarly

$$
E_{n-q}\left(\frac{G^{(q-k-1)}(x)}{(1-x)^{k+1}}\right) \leq \frac{1}{(k+1) !} E_{n-q}\left(G^{(q)}\right) .
$$

It follows, inserting these inequalities into the previous estimate, that

$$
E_{n-q}\left(\left(\frac{x G(x)}{1-x^{2}}\right)^{(q-1)}\right) \leq \frac{1}{q} \sum_{k=0}^{q-1}\left(\begin{array}{c}
q \\
k+1
\end{array}\right) E_{n-q}\left(G^{(q)}\right) .
$$

This final inequality completes the proof of the lemma.

Proof of the theorem. Let $f \in C^{q}[-1,1]$. We first note that from $f$ we can subtract a fixed polynomial $Q(x)$ of degree at most $2 q+1$ that interpolates $f^{(0)}, \ldots, f^{(q)}$ at \pm 1 , obtaining $f(x)-Q(x)=g(x)$, in which the function $g(x)$ satisfies $g^{(k)}( \pm 1)=0$ for $k=0, \ldots, q$. It is clear that for $n>2 q$ we have $E_{n-k}\left(f^{(k)}\right)=E_{n-k}\left(g^{(k)}\right)$ for $k=0, \ldots, q$. For, if $P_{n}^{(k)}$ of degree $\leq n-k$ is a polynomial of best approximation for $f^{(k)}$, then

$$
E_{n-k}\left(f^{(k)}\right)=\left\|f^{(k)}-P_{n}^{(k)}\right\|=\left\|g^{(k)}+Q^{(k)}-P_{n}^{(k)}\right\| \geq E_{n-k}\left(g^{(k)}\right) .
$$

And similarly, if a polynomial $P_{n}^{(k)}$ of degree $\leq n-k$ is a polynomial of best approximation for $g^{(k)}$ then we have $E_{n-k}\left(g^{(k)}\right)=\left\|g^{(k)}-P_{n}^{(k)}\right\|=\| f^{(k)}-$ $Q^{(k)}-P_{n}^{(k)} \| \geq E_{n-k}\left(f^{(k)}\right)$. Therefore, our theorem need only be proven for the function $g$. Now, we define the function $h(\theta)$ by $h(\theta):=g(\cos \theta) / \sin ^{q} \theta$. It follows immediately that $h(\theta)$ is a $2 \pi$-periodic function that is $q$ times continuously differentiable, and furthermore, $h(\theta), \ldots, h^{(q)}(\theta)$ are all zero at $\theta=0$ and $\theta=\pi$. Moreover, $h(\theta)$ is even if $q$ is even and odd if $q$ is odd. According to Lemma 3, we choose a polynomial of approximation $T_{n-q}(\theta)$ of order $n-q$ for $h(\theta)$ and its derivatives. It is possible as a consequence to write $T_{n-q}(\theta)=\sin ^{q} \theta Q_{n-2 q}(\cos \theta)$, in which $Q_{n-2 q}$ is a polynomial of degree at most $n-2 q$ and $Q_{n-2}(\cos \theta)$ is also zero at 0 and $\pi$. We then have

$$
\begin{aligned}
g(\cos \theta)-\sin ^{2 q} \theta Q_{n-2 q}(\cos \theta) & =\sin ^{q} \theta\left(h(\theta)-\sin ^{q} \theta Q_{n-2 q}(\cos \theta)\right) \\
& =\sin ^{q} \theta\left(h(\theta)-T_{n-q}(\theta)\right) .
\end{aligned}
$$


From this identity it follows immediately that

$$
\left|g(x)-\left(1-x^{2}\right)^{q} Q_{n-2 q}(x)\right| \leq \gamma_{0}\left(\sqrt{1-x^{2}} / n\right)^{q} E_{n-q}^{*}(h) .
$$

Furthermore, defining $H_{n}(\theta)=h(\theta)-T_{n-q}(\theta)$, it is seen by Lemma 3 that

$$
\left\|H_{n}^{(k)}(\theta)\right\| \leq\left(\gamma_{k} / n^{q-k}\right) E_{n-q}^{*}\left(h^{(q)}\right) .
$$

Therefore, by Lemma 4 , using $\varepsilon_{n}=E_{n-q}^{*}\left(h^{(q)}\right)$ and $C=\max \left\{\gamma_{0}, \ldots, \gamma_{q}\right\}$, it follows that

$$
\left\|H_{n, k}(\theta)\right\| \leq\left(C K / n^{q-k}\right) E_{n-q}^{*}\left(h^{(q)}\right),
$$

and moreover we have for $k=0, \ldots, q-1$

$$
\begin{aligned}
\frac{1}{\sin \theta}\left(\sin ^{q-k} \theta H_{n, k}(\theta)\right)^{\prime} & =\sin ^{q-k-1} \theta\left(H_{n, k}^{\prime}(\theta)+(q-k) \frac{\cos \theta H_{n, k}(\theta)}{\sin \theta}\right) \\
& =\sin ^{q-k-1} \theta H_{n, k+1}(\theta) .
\end{aligned}
$$

Consequently, we have for $k=0, \ldots, q$

$$
\left|\left(g(x)-\left(1-x^{2}\right)^{q} Q_{n-2 q}(x)\right)^{(k)}\right| \leq C \dot{K}\left(\sqrt{1-x^{2}} / n\right)^{q-k} E_{n-q}^{*}\left(h^{(q)}\right)
$$

and also $\left(g(x)-\left(1-x^{2}\right)^{q} Q_{n-2 q}(x)\right)^{(q)}=0$ at \pm 1 . Lemma 5 now shows that

$$
E_{n-q}^{*}\left(h^{(q)}(\theta)\right) \leq C \cdot K \cdot K_{q} E_{n-q}\left(g^{(q)}(x)\right) .
$$

This completes the proof of the theorem with $P_{n}(x)=\left(1-x^{2}\right)^{q} Q_{n-2 q}(x)$.

\section{REFERENCES}

1. N. Achieser and M. Krein, On the best approximation of periodic functions, Dokl. Akad. Nauk SSSR 15 (1937), 107-112. (Russian)

2. K. Balázs, T. Kilgore, and P. Vértesi, An interpolatory version of Timan's theorem, Acta Math. Hungar (to appear).

3. E. Cheney, Introduction to approximation theory, McGraw-Hill, New York, 1966.

4. J. Czipszer and G. Freud, Sur l'approximation d'une fonction périodique et de ses dérivées successives, Acta Math. 99 (1958), 33-51.

5. J. Favard, Sur les meilleures procédés d'approximation de certaines classes des fonctions par des polynômes trigonométriques, Bull. Sci. Math. (2) 61 (1937), 209-224, 243-256.

6. I. Gopengauz, $A$ theorem of A. F. Timan on the approximation of functions by polynomials on a finite segment, Mat. Z. 1 (1967), 163-172; English transl., Math. Notes 1 (1967), 110-116.

7. T. Kilgore, On simultaneous approximation, Proceedings of the Conference on Approximation Theory, Kecskemet, Hungary, August 1990 (to appear).

8. D. Leviatan, The behavior of the derivatives of the algebraic polynomials of best approximation, J. Approx. Theory 35 (1982), 169-176.

9. G. Lorentz, Approximation of functions, Holt, Rinehart, and Winston, New York, 1966.

10. S. Telyakovskii, Two theorems on the approximation of functions by algebraic polynomials, Mat. Sb. 70 (1966), 252-265.

11. A. Timan, An extension of Jackson's Theorem on the best approximation of a continuous function, Dokl. Akad. Nauk SSR 778 (1951), 17-20. (Russian)

12. R. Trigub, Approximation of functions by polynomials with integral coefficients, Izv. Akad. Nauk. SSSR Ser. Mat. 26 (1962), 261-280. (Russian)

Division of Mathematics, 120 Math Annex, Auburn University, Auburn, Alabama 36849-5307 